\title{
Prevalence and Antimicrobial Susceptibility Pattern of Streptococcus pneumoniae at General Hospital in the Central Region of Japan from December 2013 to February 2014
}

\author{
Masaaki Minami ${ }^{*}$, Ryoko Sakakibara², Taichi Imura², Hideo Morita ${ }^{2}$, Naoto Kanemaki \\ Michio Ohta ${ }^{4}$ \\ ${ }^{1}$ Department of Bacteriology, Graduate School of Medical Sciences, Nagoya City University, Nagoya, Japan \\ ${ }^{2}$ Department of Clinical Investigation, Daido Hospital, Nagoya, Japan \\ ${ }^{3}$ Department of Gastroenterology, Daido Hospital, Nagoya, Japan \\ ${ }^{4}$ School of Nursing, Sugiyama Jyogakuen University, Nagoya, Japan \\ Email: "
}

Received June 2014

\begin{abstract}
Streptococcus pneumonia infection is important cause of morbidity and mortality. This study was conducted to find out the prevalence and antimicrobial susceptibility pattern of Streptococcus pneumoniae isolates at general hospitalin the central region of Japan from December 2013 to February 2014. Streptococcus pneumoniae was identified by standard laboratory procedure. Antimicrobial susceptibility testing was performed by micro dilution assay according to CLSI recommendation. One hundred fifty-three Streptococcus pneumoniae were isolated among which $\mathbf{8 0}$ (52.2\%) were males and $73(47.8 \%)$ were females. Nasal discharge $(134 \% / 87.6 \%)$ contributed more than other biological materials. The age incidence of $(0-1)$ years, $(1-10)$ years, $(11-40)$ years, $(41-60)$ years and $>60$ years age groups were $26(17.0 \%), 110(71.9 \%), 3(2.0 \%), 10$ (6.5\%), and $4(2.6 \%)$ respectively. Positive samples were received mostly from the pediatrics $(137 \% / 89.5 \%)$, respiratory medicine $(12 \% / 7.8 \%)$ and lowest from gastroenterology $(1 \% / 0.6 \%)$ and neurology (1/ $0.6 \%)$ department. Vancomycin and rifampicin were the most active antibiotics with $100 \%$ susceptibility rates. The next best were levofloxacin, penicillin $G$ and ceftriaxone. Our study revealed that 82 Streptococcus pneumonia isolates had multidrug resistant ability (53.6\%). Streptococcus pneumoniae infection spreads among community easily and inappropriate use of antibiotics contributes to their resistance. Continuous antimicrobial susceptible surveys are essential to guide policy on the adequate use of antibiotics to reduce the morbidity and mortality and reduce the emergency of antimicrobial resistance.
\end{abstract}

\section{Keywords}

Streptococcus pneumoniae, Susceptibility, Antimicrobial Resistance, Multi Drug Resistance

\footnotetext{
${ }^{*}$ Corresponding author.

How to cite this paper: Minami, M., et al. (2014) Prevalence and Antimicrobial Susceptibility Pattern of Streptococcus pneumoniae at General Hospital in the Central Region of Japan from December 2013 to February 2014. Journal of Biosciences and Medicines, 2, 12-17. http://dx.doi.org/10.4236/jbm.2014.26003
} 


\section{Introduction}

Streptococcus pneumoniae is one of the most common bacteria causing pneumonia, meningitis, bacteremia and many other infections, and continues to be an important cause of morbidity and mortality in the world [1].

Globally, an estimated 1.6 million people including 1 million children less than 5 years old die of invasive pneumonia disease annually [2]. Its clinical burden is concentrated among the very old and very young [2]. Despite this high toll, the vast majority of Streptococcus pneumoniae are found in asymptomatic nasopharyngeal carriage, the prevalence of which varies by age and region [3]. The carriage state is responsible for transmission, and is the stage of Streptococcus pneumoniae life history at which interventions such as antibiotics and vaccines exert their selective pressure [4].

The present study was conducted to find out the recent prevalence and antimicrobial susceptibility pattern of Streptococcus pneumoniae isolates at general hospital in the central of Japan. Our result would be useful in establishing empiric therapy guidelines and to contribute to larger more extensive surveillance study.

\section{Materials and Methods}

\subsection{Strains and Clinical Data Collection}

A total of 153 Streptococcus pneumoniae were obtained from various clinical specimens at Daido Hospital from December 2013 to February 2014. Daido Hospital is a 404-bed private general hospital in the central region of Japan. We used medical records appended to clinical species for the analysis of clinical feature at Daido Hospital. We considered several isolates from the same region of the same patient as one isolate per one patient for the analysis in this study. All streptococcal isolates were identified by standard conventional biochemical methods or the VITEK2 system (bioMérieux, Durham NC, USA). Our experimental design was approved by the ethics committee at Daido hospital.

\subsection{Antimicrobial Susceptibility Analysis}

Streptococcus pneumoniae isolates were examined for 15 antibiotic susceptibilities as follows; PCG, penicillin G; AMPC, amoxicillin; CTX, cefotaxime; CTRX, ceftriaxone; IPM, imipenem; MEPM, meropenem; LVFX, levofloxacin; OFLX, ofloxacin; CAM, clarithromycin; EM, erythromycin; VCM, vancomycin; TC, tetracycline; CP, chloramphenicol; RFP, rifampicin, ST, Trimethoprim-sulfamethoxazole. Minimal inhibitory concentration (MICs) were determined at clinical laboratory in Daido Hospital using broth micro dilution methodology with the VITEK2 system. MICs were calculated as MIC $_{90}$ (MIC causing inhibition of $90 \%$ of isolates). Percentage susceptibilities were calculated based on Clinical Laboratory Standard Institute (CLSI) break point [5]. We used two separate interpretive breakpoints for meningeal and non-meningeal isolates to define penicillin. Multidrug resistance (MDR) was defined as non-susceptibility to more than any three antimicrobial agents [6].

\subsection{Statistical Analysis of the Data}

We conducted the statistical analysis with the chi-squared test or Fisher's exact test when appropriate. Differences were considered significant when $p$ was $<0.05$.

\section{Results}

One hundred fifty-three Streptococcus pneumoniae were isolated among which 80 (52.2\%) were from male patient and 73 (47.8\%) were from females. Nasal discharge 134 (87.6\%) [male-69, female-65], sputum 15 (9.7\%) [male-9, female-6], tonsil 2(1.3\%) [male-1, female-1], pharyngeal mucus 1(0.7\%) [male-1, female-1], and blood $1(0.7 \%)$ [male-1, female-1] were the source of Streptococcus pneumoniae isolates (Table 1). There was no significant difference between male and female.

The age incidence among 0 - 1 years age group was 26 (17\%) [male-17, female-9], among 1 - 10 years age group, $110(71.9 \%)$ [male-52, female-58] ( $p<0.05)$, among $11-40$ years age group, $3(1.7 \%)$ [male-2, female-1], in 41-60 years it was 10 (6.5\%) [male-8, female-2] and in $>60$ years age group it was 4 (2.6\%) [male-2, female-2] (Table 2). There was no significant difference between male and female.

Most of the Streptococcus pneumoniae isolates were from the pediatrics (137/89.5\%) [male-69, female-68] followed by respiratory medicine (12/7.8\%) [male-9, Female-3], general medicine (2/1.3\%) [male-1, female-1] 
and lowest from gastroenterology (1/0.6\%) [male-0, female-1], and neurology department (1/0.6\%) [male-1, female-0] (Table 3). There was no significant difference between male and female.

The results of antimicrobial susceptibility of Streptococcus pneumoniae isolates to various antibiotics tested in this study are shown in Table 4. Vancomycin and rifampicin were the most active antibiotics with $100 \%$.

Table 1. Biological material wise distribution of Streptococcus pneumoniae isolates.

\begin{tabular}{cccccc}
\hline biological material & nasal discharge & sputum & tonsil & pharyngeal mucus \\
\hline male & 69 & 9 & 1 & 0 & 1 \\
female & 65 & 6 & 1 & 1 & 1 \\
total & 134 & 15 & 2 & 1 \\
\hline
\end{tabular}

Table 2. Age wise distribution of Streptococcus pneumoniae isolates.

\begin{tabular}{cccccc}
\hline age groups & $(0-1)$ years & $(1-10)$ years & $(11-40)$ years & $(41-60)$ years & $>60$ years \\
\hline male & 17 & 52 & 1 & 8 & 2 \\
female & 9 & 58 & 2 & 2 & 2 \\
total & 26 & 110 & 3 & 10 & 4 \\
\hline
\end{tabular}

Table 3. Clinical department wise distribution of Streptococcus pneumoniae isolates.

\begin{tabular}{|c|c|c|c|c|c|}
\hline clinical department & pediatrics & respiratory medicine & general medicine & gastroenterology & neurology \\
\hline male & 69 & 9 & 1 & 0 & 1 \\
\hline female & 68 & 3 & 1 & 1 & 0 \\
\hline total & 137 & 12 & 2 & 1 & 1 \\
\hline
\end{tabular}

Table 4. Antimicrobial susceptibility of Streptococcus pneumoniae isolates.

\begin{tabular}{cccc}
\hline antimicrobial agents & MIC90 $(\mathrm{mg} / \mathrm{mL})$ & range & \% susceptibility \\
\hline PCG & 2 & $\leq 0.06-4$ & 97.4 \\
AMPC & 2 & $\leq 0.06-4$ & 92.8 \\
CTX & 1 & $\leq 0.06-4<$ & 93.5 \\
CTRX & 1 & $\leq 0.06-4<$ & 74.5 \\
IPM & 0.25 & $<0.03-0.5$ & 73.9 \\
MEPM & 1 & $\leq 0.06-0.12$ & 98.7 \\
LVFX & 2 & $\leq 0.5-8 \leq$ & 88.9 \\
OFLX & 4 & $\leq 1-8 \leq$ & 6.5 \\
CAM & $1 \leq$ & $\leq 0.25-1 \leq$ & 7.2 \\
EM & $1 \leq$ & $\leq 0.25-1 \leq$ & 100.0 \\
VCM & $\leq 1$ & $\leq 1$ & 8.5 \\
TC & $16 \leq$ & $\leq 1-16 \leq$ & 87.6 \\
CP & 16 & $\leq 2-32 \leq$ & 100.0 \\
RFP & $\leq 0.25$ & $\leq 0.25$ & 79.7 \\
ST & 20 & $\leq 10-80$ &
\end{tabular}


susceptibility rates. The next best antibiotics with over 95\% susceptibility rates were levofloxacin (98.7\%), penicillin G (97.4\%), and ceftriaxone (96.1\%). Significant resistant were observed in tetracycline (8.5\%), erythromycin (7.2\%), clarithromycin (6.5\%).

Our study revealed that 82 Streptococcus pneumoniae isolates had multidrug resistant ability (53.6\%). The most common pattern of MDR was resistant to clarithromycin, tetracycline, and imipenem (25.5\%), followed by resistant to clarithromycin, tetracycline, and trimethoprim - sulfamethoxazole (16.3\%). Most Streptococcus pneumoniae isolates with MDR were resistant to at least one of the macrolides tested.

\section{Discussion}

In this study, we described the characteristics of Streptococcus pneumonia isolates from December 2013 to February 2014 at general hospital in the central region of Japan.

With respect to gender group, Streptococcus pneumoniae were isolated more from female patients than male patients. Although previous report showed that the number of male patient was significant greater than that of female patient [7], our study showed the male to female ratio was about 1.1 time and there was no significant differences among gender.

We clarified Streptococcus pneumoniae with age distribution. The present study reveals the prevalence of Streptococcus pneumonia more in extremes of age group as seen in 0 - 1 years age group, it is $17 \%$, increasing to $71.9 \%$ in $1-10$ years age group, diminishing to $2 \%$ in 11 - 40 years age group, again increasing to $6.5 \%$ in $41 \%$ - 60 years age group and finally diminished to $2.6 \%$ in more than 60 years age group. Although young patients under 10 years frequently caused Streptcococcus pneumoniae infection, the large numbers of Streptcococcus pneumoniae were not isolated from over 60 years age patients in our study. Previous report showed that high incidence of pneumonia in children and elder people in different parts of world [7] [8]. It is suggested to decrease immunity in the extremes of age groups.

In the analysis of clinical departments, we found that department where most patients with Streptococcus pneumoniae were detected was pediatrics. Streptococcus pneumoniae infection, especially acute pharyngitis and tonsillitis, was usually popular as pediatric diseases [1].

The disease burden of Streptococcus pneumoniae infections has increased due to widespread emergence of antimicrobial resistance in many countries from 1990's [2]. Previous studies documented very high prevalence rates of beta-lactam and macrolide resistance in Streptococcus pneumoniae in Asian countries [2] [9]-[13]. Particularly, macroride resistance has remarkably increased in many Asian countries, where over $70 \%$ of clinical isolates were fully resistant [2] [9] [12] [13]. Antimicrobial susceptible analysis of Streptococcus pneumoniae revealed that macrolide was no longer effective against these bacteria because macrolide resistant rates of Streptococcus pneumoniae were more than 90\%. Recently Clarithromycin and Azithromycin, which we call new macrolide, have been used frequently against respiratory tract infectious disease. Thus, we need further antimicrobial surveillance to prevent the spread of new macroride resistant Streptococcus pneumoniae.

Streptococcus pneumoniae also had tetracycline - resistant pattern. This result implies that Streptococcus possesses the same pathogenic island including both macrolide and tetracycline resistant genes [4]. We found a low prevalence of penicillin resistance in non-meningeal isolates according to the revised CLSI breakpoints for resistance to parenteral penicillin, although penicillin MICs have increased in some countries such as China and India compared with past investigation [9]. Most of the non-meningeal isolates from Asian countries were susceptible to parenteral penicillin, a finding consistent with other study worldwide $(<7 \%)$ [2] [9] [13]. As the emergence of penicillin resistant Streptococcus pneumoniae is low, our result showed that the prevalence of imipenem non-susceptibility was not low in Japan. We need to focus on the carbapenem susceptible pattern in Streptococcus pneumonia hereafter. The overall rates of resistance to fluoroquinolone in Streptococcus pneumoniae remained low in most countries including Japan [2] [9] [13]. Thus, given the popular use of respiratory fluoroquinlone in clinical practice, the emergency of these strains highly resistant to fluoroquinolone could be a concern in the future in the treatment of Streptococcus pneumoniae pneumonia. In addition, MDR Streptococcus pneumonia was also very prevalent in Asian countries (59.3\%), particularly in China (83.3\%), Vietnam (75.5\%), South Korea (63.9\%), Hong Kong (62.2\%), and Taiwan (59.7\%) [9]. From our result, multi drug resistance was $53.6 \%$ in Japan. But this rate in Japan was much higher than those in other parts world such as $9 \%$ to $24 \%$ in North America and $0 \%$ to 43\% in Europe [2] [9] [14]. The emergence of MDR Streptococcus pneumonia including macrolide, tetracycline, and carbapenem resistant strains will raise the serious problem in Asian coun- 
tries including Japan [13] [15].

\section{Conclusions}

Incidence of Streptococcus pneumoniae infection is increasing worldwide affecting the children and aged adult population which may lead to severe invasive infection by dissemination to other organs of the body if not treated adequately. The indiscriminate and inadvertent use of antibiotics has led to the emergence of multidrug resistance among commonly used antibiotics.

Our investigation aims to guide medical officer on appropriate use of antibiotics. This aim is not only to reduce the morbidity and mortality in the patients but also to control the emergence and spread of resistance among Streptococcus pneumoniae. Continuous surveillance of the use of antibiotics helps in preserving the effectiveness of antibiotics. The results from our study strongly emphasize the need for continuous epidemiological monitoring of antibiotic resistant.

\section{Acknowledgements}

We thank Mr. Shoji Ishihara and Ms. Miwako Fujimura for special encouragement. This study was supported by a grant-in-aid for research from the Nagoya City University, Japan.

\section{References}

[1] Mitchell, A.M. and Mitchell, T.J. (2010) Streptococcus pneumoniae: Virulence Factors and Variation. Clinical Microbiology and Infection, 16, 411-418. http://dx.doi.org/10.1111/j.1469-0691.2010.03183.x

[2] Lynch, J.P. and Zhanel, G.G. (2009) Streptococcus pneumoniae: Epidemiology, Risk Factors and Strategies for Prevention. Seminars in Respiratoryand Critical, Care Medicine, 30, 189-209. http://dx.doi.org/10.1055/s-0029-1202938

[3] Crook, D.W., Brueggemann, A.B., Sleeman, K.L. and Peto, T.E.A. (2004) Pneumococcal Carriage. In: Tuomanen, E.I., Mitchell, T.J., Morrison, D.A. and Spratt, B.G., Eds., The Pneumococcus, American Society for Microbiology, Washington DC, 136-148.

[4] Willems, R.J.L., Hanage, W.P., Bessen, D.E. and Feil, E.J. (2011) Population Biology of Gram-Positive Pathogens: High-Risk Clones for Dissemination of Antibiotic Resistance. FEMS Microbiology Review, 35, 872-900. http://dx.doi.org/10.1111/j.1574-6976.2011.00284.x

[5] Clinical and Laboratory Standards Institute (CLSI) (2014) Performance Standards for Antimicrobial Susceptibility Testing: 24st Informational Supplement. Clinical and Laboratory Standards Institute M100-S24, Wayne.

[6] Magiorakos, A.P., Srinivasan, A., Carey, R.B., Carmeli, Y., Falagas, M.E., Giske, C.G., et al. (2012) Multidrug-Resistant, Extensively Drug-Resistant and Pandrug-Resistant Bacteria: An International Expert Proposal for Interim Standard Definitions for Acquired Resistance. Clinical Microbiology and Infection, 18, 268-281. http://dx.doi.org/10.1111/j.1469-0691.2011.03570.X

[7] Geng, Q., Zhang, T., Ding, Y., Tao, Y., Lin, Y., Wang, Y., et al. (2014) Molecular Characterization and Antimicrobial Susceptibility of Streptococcus pneumoniae Isolated from Children Hospitalized with Respiratory Infections in Suzhou, China. PLoS One, 9, e93752. http://dx.doi.org/10.1371/journal.pone.0093752

[8] Hofman, J., Cetron, M.S. and Farley, M.M. (1995) The Prevalence of Drug Resistant Streptococcus pneumoniae in Atlanta. New England. Journal of Medicine, 333, 481-486. http://dx.doi.org/10.1056/NEJM199508243330803

[9] Kim, S.H., Song, J.H., Chung, R.D., Thamlikitkul, V., Yang, Y., Wang, H., et al. on Behalf of ANSORP Study Group (2012) Changing Trends in Antimicrobial Resistance and Serotypes of Streptococcus pneumoniae Isolates in Asian Countries: An Asian Network for Surveillance of Resistant Pathogens (ANSORP) Study. Antimicrobial Agents and Chemotherapy, 56, 1418-1426.

[10] Xue, L., Yao, K., Xie, G., Zheng, Y., Wang, C., Shang, Y., et al. (2010) Serotype Distribution and Antimicrobial Resistance of Streptococcus pneumoniae Isolates That Cause Invasive Disease among Chinese Children. Clinical Infectious Disease, 50, 741-744. http://dx.doi.org/10.1086/650534

[11] Zhao, C., Zhang, F., Chu, Y., Liu, Y., Cao, B., Chen, M., et al. (2013) Phenotypic and Genotypic Characteristic of Invasive Pneumococcal Isolates from Both Children and Adult Patients from a Multicenter Surveillance in China 2005-2011. Plos One, 8, e82361. http://dx.doi.org/10.1371/journal.pone.0082361

[12] Zhang, Y., Chen, Y., Wang, Z., Li Y., Wang, D., Shang, Y., et al. (2013) Serological and Molecular Capsular Typing, Antibiotic Susceptibility and Multilocus Sequence Typing of Streptococcus pneumoniae Isolates from Invasive and Non-Invasive Infections. Chinese Medical Journal, 126, 2296-2303.

[13] Okade, H., Funatsu, T., Eto, M., Furuya, Y., Mizunaga, S., Nomura, N., et al. (2014) Impact of the Pneumococcal 
Conjugate Vaccine on Serotype Distribution and Susceptibility Trends of Pediatric Non-Invasive Streptococcus pneumoniae Isolates in Tokai, Japan over a 5-Year Period. Journal of Infection and Chemotherapy. http://dx.doi.org/10.1016/j.jiac.2014.03.010

[14] Dunais, B., Bruno-Bazureault, P., Carsenti-Dellamonica, H., Touboul, P. and Pradir, C. (2011) Adecade-Long Surveillance of Nasopharyngeal Colonization with Streptococcus pneumoniae among Children Attending Day-Care Centres in South-Eastern France: 1999-2008. European Journal of Clinical Microbiology and Infection Disease, 30, 837-843. http://dx.doi.org/10.1007/s10096-011-1154-9

[15] Tajima, T., Sato, T., Toyonaga, Y., Hanaki, H. and Sunakawa, K. (2013) Nationwide Survey of the Development of Drug-Resistant Pathogens in the Pediatric Field in 2007 and 2010: Drug Sensitivity of Streptococcus pneumoniae in Japan (Second Report). Journal of Infection and Chemotherapy, 19, 510-516. http://dx.doi.org/10.1007/s10156-013-0593-x 
Scientific Research Publishing (SCIRP) is one of the largest Open Access journal publishers. It is currently publishing more than 200 open access, online, peer-reviewed journals covering a wide range of academic disciplines. SCIRP serves the worldwide academic communities and contributes to the progress and application of science with its publication.

Other selected journals from SCIRP are listed as below. Submit your manuscript to us via either submit@scirp.org or Online Submission Portal.
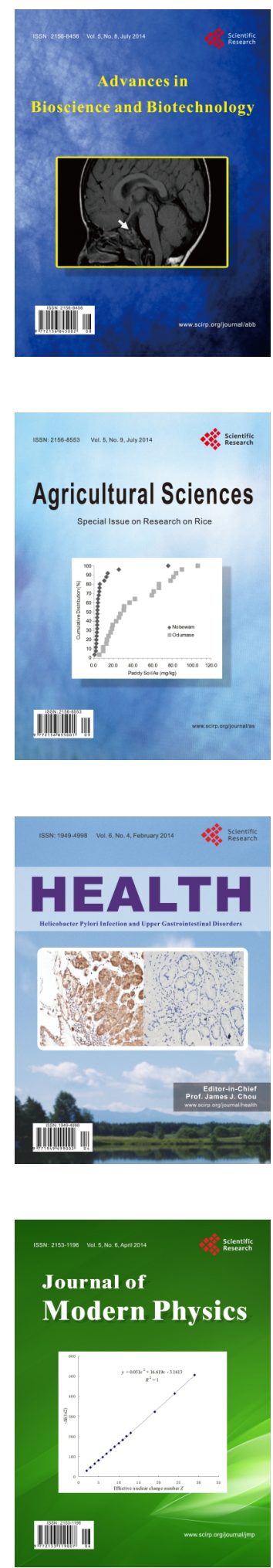
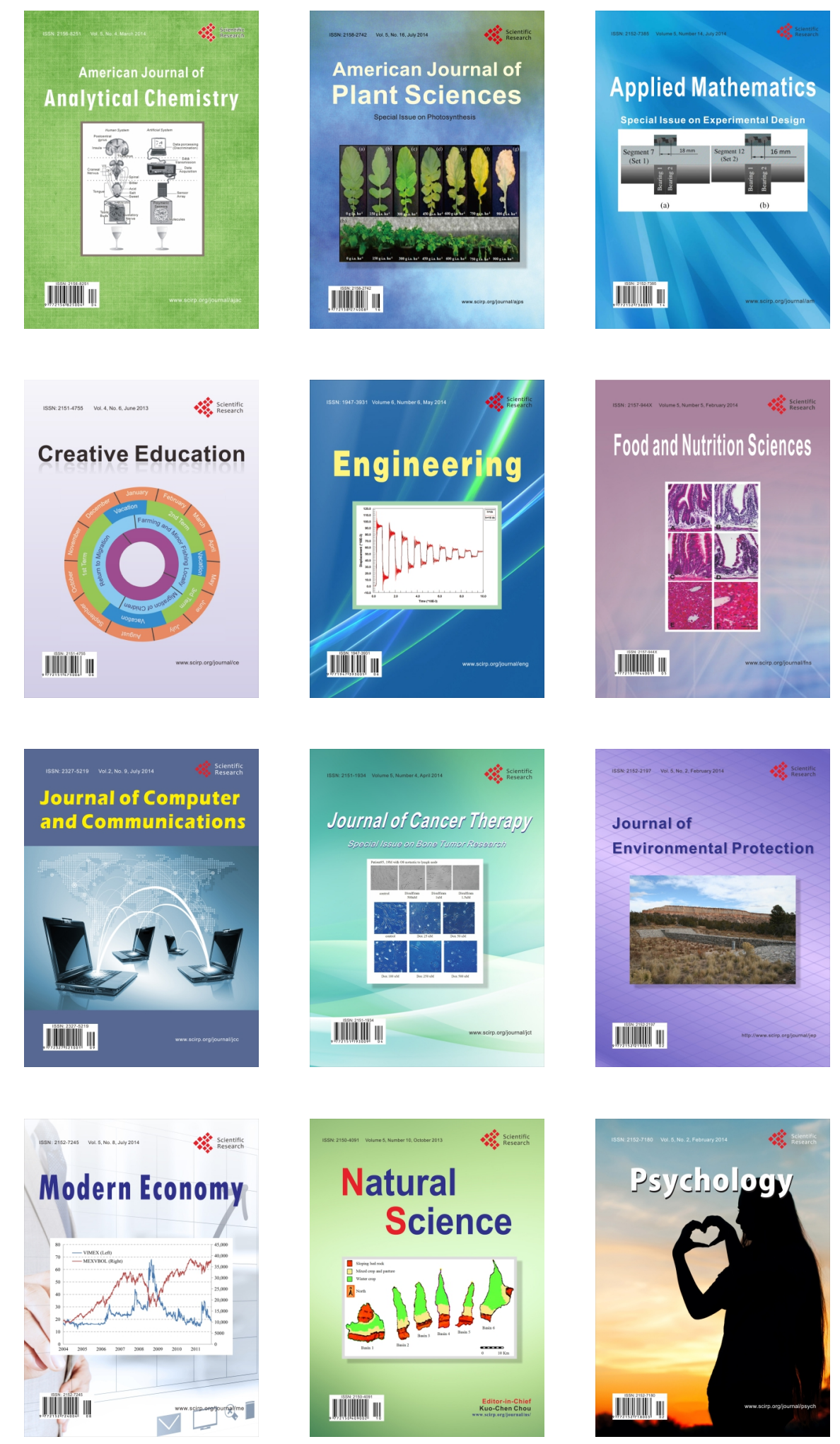